\title{
Condiciones geodinámicas derivadas del terremoto y tsunami de 2010 en la costa de Chile central. El caso de Pichilemu ${ }^{1}$
}

\author{
María-Victoria Soto ${ }^{2}$, Joselyn Arriagada ${ }^{3}$, Carmen Paz Castro-Correa ${ }^{4}$, \\ Ignacio Ibarra ${ }^{5}$ y Giuliano Rodolfi ${ }^{6}$
}

\begin{abstract}
RESUMEN
El terremoto y tsunami de 2010 generó cambios en la morfología y dinámica sedimentaria del litoral de Pichilemu, Chile central, evidenciado a través del retroceso y erosión de las playas arenosas y dunas. Se identificaron los patrones de modificación a través del análisis de imágenes satelitales anteriores y posteriores al terremoto/tsunami mencionado, comparando con registros geomorfológicos del año 2004. Se realizó trabajo de terreno para la observación de formas esenciales y medición de perfiles de playa representativos de la dinámica de las ensenadas estructurales (headland bay beach); se analizó la variabilidad del tipo de playa dominada por olas. Los resultados indican que existe erosión litoral y un cambio en el balance sedimentario asociado a la subsidencia de la costa, como también, que transcurridos cuatro años del evento extremo, luego de la fase erosiva, se observa un cambio tendiente hacia la progradación del litoral arenoso siguiendo el patrón dinámico típico de sistemas de ensenadas estructurales.
\end{abstract}

Palabras Clave: Ensenada; tsunami; microdunas; cambios en la playa

\begin{abstract}
The earthquake and tsunami of 2010, triggered changes in the geomorphological and sedimentary dynamics along the coast of Pichilemu, in central Chile. These changes were evidenced through the retreat and erosion of sandy beaches and coastal sand dunes. Modification patterns were identified through analysis of satellite images prior to and after the earthquake/tsunami, and compared with records from geomorphological fieldwork carried out in 2004 that measured essential features of representative beach profiles in the headland bay beaches. The variability of beaches dominated by waves was analyzed. The results indicate that there is coastal erosion and a change in the sedimentary balance associated with the subsidence of the coast, and that four years after the earthquake/tsunami, after the erosive phase, a change in tendency towards progradation of sandy coastlines in the headland bay beaches can be observed
\end{abstract}

Key words: Headland bay beach; tsunami; micro dune, beach changes

1 Fondecyt $\mathrm{N}^{\circ}$ 110022. Artículo recibido el 31 de diciembre de 2013, aceptado el 13 de agosto de 2014 y corregido el 3 de octubre de 2014.

2 Departamento de Geografía, Universidad de Chile (Chile).E-mail: mvsoto@uchilefau.cl

3 Departamento de Geografía, Universidad de Chile (Chile). E-mail: joarriag@uchile.cl

\footnotetext{
4 Departamento de Geografía, Universidad de Chile (Chile).E-mail: cpcastro@uchilefau.cl

5 Departamento de Geografía, Universidad de Chile (Chile).E-mail: ieibarra@uchilefau.cl

6 Universidad de Florencia (Italia). E-mail: giurodolfi@yahoo.it
} 
El terremoto y tsunami que afectó a la zona centro-sur de Chile el 27 de febrero de 2010 (evento conocido como 27F) generó un gran impacto debido a la pérdida de vidas humanas, de propiedad privada y pública y al significativo daño en la infraestructura de la zona. En el área costera el tsunami afectó los asentamientos poblados, siendo los más dañados aquellos en que habitan las comunidades de pescadores artesanales (Castilla, 1988; Castilla et al., 2010), cuyas fuentes laborales también se vieron afectadas, en algunos casos hasta los días actuales. Sin embargo, estos impactos no solo pueden ser evaluados en sus aspectos sociales y económicos, sino que también en impactos en el sistema físiconatural, donde se pueden evaluar las modificaciones en la morfología y dinámica costera de los litorales arenosos, en que recién al cabo de cuatro años se observan evidencias de un retorno a las condiciones existentes antes del evento tectónico.

En este escenario de cambios, cobra relevancia la dinámica exógena de las unidades básicas del paisaje costero, tales como las playas arenosas, las dunas y el litoral cercano, ya que las formas y procesos que caracterizan la línea de costa están fuertemente relacionadas con su exposición a los agentes oceanográficos que explican la condición de cambio de las playas arenosas (ArayaVergara, 1983; 1985; 1986; Martínez, 2001; Soto, 2005; Soto \& Arriagada, 2007; Soto et al., 2010; Arriagada 2009; Arriagada et al., 2014)

La dinámica litoral en la zona de Pichilemu (Figura $\mathrm{N}^{\circ} 1$ ), Chile central $\left(34^{\circ} 40^{\prime}\right.$ $\left.\mathrm{S} / 72^{\circ} 00^{\prime} \mathrm{W}\right)$ derivada del evento sísmico del $27 \mathrm{~F}$, representa una respuesta cosísmica de carácter local que explica los procesos actuales en el marco de una costa de control estructural con dominio de ensenadas controladas por la estructura (headland bay beach).

La influencia tectónica y estructural de esta sección de la costa chilena, condicionan la posición relativa de la línea de costa, la dinámica del oleaje, los tipos de playa dominadas por olas existentes y consecuentemente, los procesos geodinámicos dominantes en la zona litoral (Araya-Vergara, 1983; 1985; 1986; 1996, 2003).
En este contexto, es importante conocer las características particulares de la tectónica regional, ya que en Pichilemu se había identificado una brecha sísmica (PichilemuConstitución), a la que se asociaba la generación potencial de un futuro terremoto, con intensidad estimada de Mw 8.1 (Comte et al., 1986; Nishenko, 1991, González, 1997). Por su parte, Ruegg et al. (2009) estimaron que la brecha sísmica Constitución-Concepción podría generar un evento sísmico similar. El 27 de febrero de 2010 ocurrió el terremoto interplacas de magnitud $8.8 \mathrm{Mw}$, que abarcó la zona costera del sur de Chile, con un epicentro en la ciudad de Cobquecura, localización indicada por Farías et al. (2010) como el área de la brecha sísmica. Otro evento sísmico importante sucedió el 11 de marzo del 2010, con epicentro en la costa de Pichilemu (7.0 Mw) asociado a la Secuencia Sísmica de Pichilemu, atribuido a la reactivación de faIlas de orientación NW-SE, indicativo de una ruptura cortical (Farías et al., 2011).

El evento $27 \mathrm{~F}$, con un área de influencia de más de 500 km, generó condiciones tanto de subsidencia como de alzamiento cosísmico en el área costera de Chile centro sur, y un tsunami de gran impacto en el borde costero de las regiones del Biobío, Maule, O'Higgins y Valparaíso (Vargas et. al., 2011). La altura de la ola de tsunami en las localidades de Pichilemu y Cáhuil fue estimada entre los 4 y 6 m (Lagos, 2012).

En Pichilemu, el efecto cosísmico registrado por Farías et al. (2010) fue el solevantamiento de $20 \mathrm{~cm}$. A su vez Quezada et al. (2010) indican para el mismo sector un movimiento cosísmico vertical neto de -35 cm (considerando los eventos sísmicos del 27 de febrero y 11 de marzo del 2010). El evento del 11 de marzo habría generado una subsidencia neta del orden de los 0,5 m (comunicación verbal de Marcelo Farías, 2012).

El análisis de los impactos cosísmicos y del tsunami en la costa de Pichilemu puede ser comprendido en el contexto de la dinámica litoral en las ensenadas o bahía de control estructural presentes en gran parte de Chile, y en especial es esta zona. Las ensenadas estructurales (headland bay beaches) son consideradas sistemas complejos, cuya génesis está 
Figura $\mathrm{N}^{\circ} 1$

Área de estudio (óvalos y su numeración indican localización de las figuras en el texto, del análisis multitemporal)

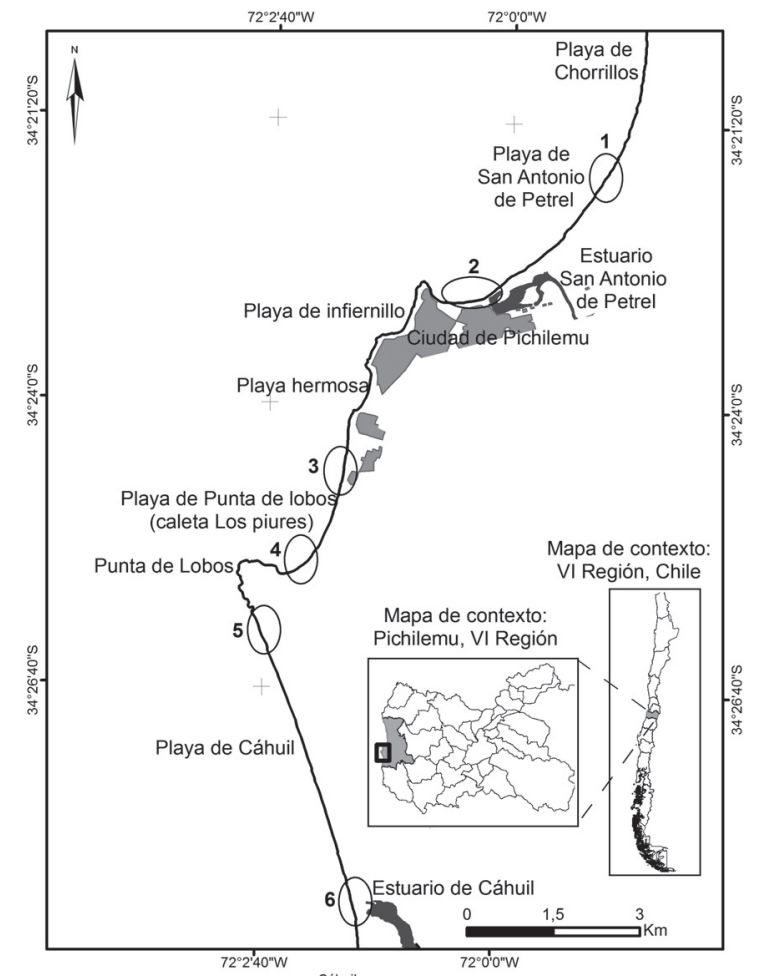

Fuente: Elaboración propia.

asociada a la conformación morfotectónica y estructural del litoral (Le Roux et al., 2006).

En estos sistemas litorales, David (1982) destacó la importancia de la refracción de las olas en la dinámica morfosedimentológica. La forma de bahías en espiral logarítmica fue reconocida como tal por Yasso (1965), y posteriormente tratada por Le Blond (1979) y Lavalle \& Lakhan (1997). Hsu \& Evans (1989) desarrollaron la teoría de las formas parabólicas en bahías. A partir de estos conceptos, las bahías han sido también tratadas como headland bay beach, de acuerdo a autores como Short (1999), Sweers (1999), Bird (2000); Benedet et al. (2004), Klein \& De Menezes (2001), Klein et al. (2002b); Benedet et al. (2004), Komar (2010), Klein et al. (2010), Simeone \& De Falco (2013).
En Chile, las bahías y ensenadas han sido estudiadas por Araya-Vergara (1983, 2003), usando la conceptualización de bahías en forma de zeta o de medio corazón, según Silvester o de espiral logarítmica (Yasso, 1965; Krumbein, 1947).

En estas playas, la presencia y el efectos de protección del headland o saliente rocosa, conlleva a una distribución sistemática de la energía del oleaje en dirección deriva abajo, desde la zona proximal a la distal por el efecto de la saliente rocosa (Araya-Vergara, 1983; Martínez, 2001; Martínez et al., 2011; Soto, 2005; Villagrán, 2007; Soto y Arriagada, 2007; Soto et al., 2010; Arriagada et al., 2014). La saliente rocosa corresponde a remanentes de erosión diferencial, controlados por la estructura y la litología. 
La acción del oleaje es fundamental en la dinámica sedimentaria a lo largo del sistema de ensenadas y es evaluado a través de la caracterización de la zona de surf. La variabilidad espacial y temporal de las formas asociadas de la playa se relaciona con la dinámica del oleaje, las corrientes de la zona de rompiente y el transporte de sedimentos (Araya-Vergara, 1983; Aagaard \& Masselink, 1999; Aagaard \& Hughes, 2006; Aagaard et al., 2004; Masselink, 1999; Masselink et al., 2005; Brander \& Short, 2000; Elgar et al., 2001; Aagaard et al., 2004).

Consecuente con los antecedentes anteriores, el propósito de esta investigación es identificar el impacto asociado al terremoto y tsunami del 27 febrero de 2010 en las ensenadas de Pichilemu, Punta de Lobos y Cáhuil, analizando los cambios ocurridos en la morfología litoral a nivel de playas arenosas y dunas, a través de la caracterización de la dinámica litoral y la tendencia evolutiva del sistema post evento 27F.

\section{Metodología}

Contexto tectónico y morfoestructural: a través de los trabajos clásicos de Brüggen (1950), Ceccioni (1970) y Araya-Vergara (1983), se analizó el escenario morfotectónico y estructural del área de estudio. Estos autores destacaron la génesis de las terrazas marinas elevadas, las terrazas del Mioceno y la morfogénesis alineada de la línea de costa, respectivamente. A partir de esta información se generó una carta geomorfológica, complementada con interpretación de fotos aéreas (desde 1962 a 2005) y el trabajo de terreno. La información fue actualizada a los años 2010 y 2013 con el objeto de identificar los impactos en la geodinámica asociados al terremoto y tsunami en la zona costera.

Geomorfología costera: la línea de costa fue analizada desde la perspectiva teórica y conceptual de las bahías controladas por la estructura (Short, 1999). Se realizaron perfiles de playa en terreno a través del método de Emery (1961), los que fueron comparados con aquellos realizados en años anteriores (Soto, 2005). Se aplicó la taxonomía de perfiles de playa de Araya-Vergara (1986). Estos perfiles consideran desde la zona de máxima resaca hasta la zona de dunas anteriores. Se identificaron las formas dunarias existentes, aplicando las nociones del continuum dunar (Araya-Vergara, 1996. En: Arriagada, 2009).

Se identificaron los patrones de cambio espacio-temporal de los procesos dinámicos y de las formas esenciales de los sistemas playa-duna, mediante el análisis multitemporal de imágenes satelitales Google Earth disponibles de fechas anteriores y posteriores al evento (2004, 2007,2008, 2010 y 2014), las cuales fueron georreferenciadas y rectificadas en el software ArcGis 10.1 (con un error RMS de 0,7$)$.

Dinámica de la zona de rompiente: los estudios realizados en Autralia por Short (1999, 2005), en Brasil por Klein \& De Menezes (2001), Klein et al. (2002a), Klein \& Schumacher (2002), y en Chile por Araya-Vergara (1996) Martínez (2001), Martínez et al. (2011); Soto (2005), Soto \& Arriagada (2007), Soto et al. (2010), dan cuenta de la importancia de la hidrodinámica costera en las bahías de origen estructural.

A partir de la condición conocida de subsidencia en el litoral de Pichilemu, se buscó las evidencias del cambio cosísmico en la zona de rompiente. Para ello se realizaron observaciones de campo aplicando la taxonomía de Wright \& Short (1984, En: Short, 1999), de playas dominadas por olas (playas reflectivas, disipativas, intermedias) y se comparó con los resultados obtenidos en años anteriores. El análisis temporal fue realizado a través del análisis de fotos aéreas e imágenes satelitales (1962-1963, 1978, 1994, 1997, 1998, 2003, 2004, 2006, 2007, 2008, 2009, 2010, 2011, 2012 y 2014) y el trabajo de campo de marzo y julio del 2004, enero y julio del 2005, 2009, marzo de 2011, enero y julio de 2012 y enero 2013.

\section{Evidencias morfológicas de cambio en las playas y dunas posterremoto y tsunami:}

A partir de la elaboración de la carta geomorfológica y la consideración de los cambios en la playa de los principales grupos de formas, se analizaron los cambios atribuidos a consecuencias cosísmicas e inundación del 
tsunami (Figura $\mathrm{N}^{\circ} 2$ ). En las playas arenosas se evidenció el retroceso de las playas y la desctrucción parcial de los cordones de dunas anteriores. En la ensenada de Pichilemu, en trabajo anterior de Soto (2005) se había identificado hasta cuatro cordones de dunas anteriores (parte distal), al igual que la presencia de dunas embrionarias; post-tsunami solo es posible identificar un cordón de dunas en estado de semidestrucción.
Es importante señalar que en relación a las dunas propiamente tales, el mayor espesor se encuentra en la parte media de la bahía de Pichilemu, conformando un sistema de dunas transgresivas que cabalgan el escarpe y la terraza marina; en el año 1962 era un campo de dunas libres que actualmente está totalmente forestado. En Punta de Lobos y Cáhuil existe un pequeño campo de dunas transgresivas con presencia de formas longitu-

Figura $\mathrm{N}^{\circ} 2$

Carta geomorfológica del área de estudio

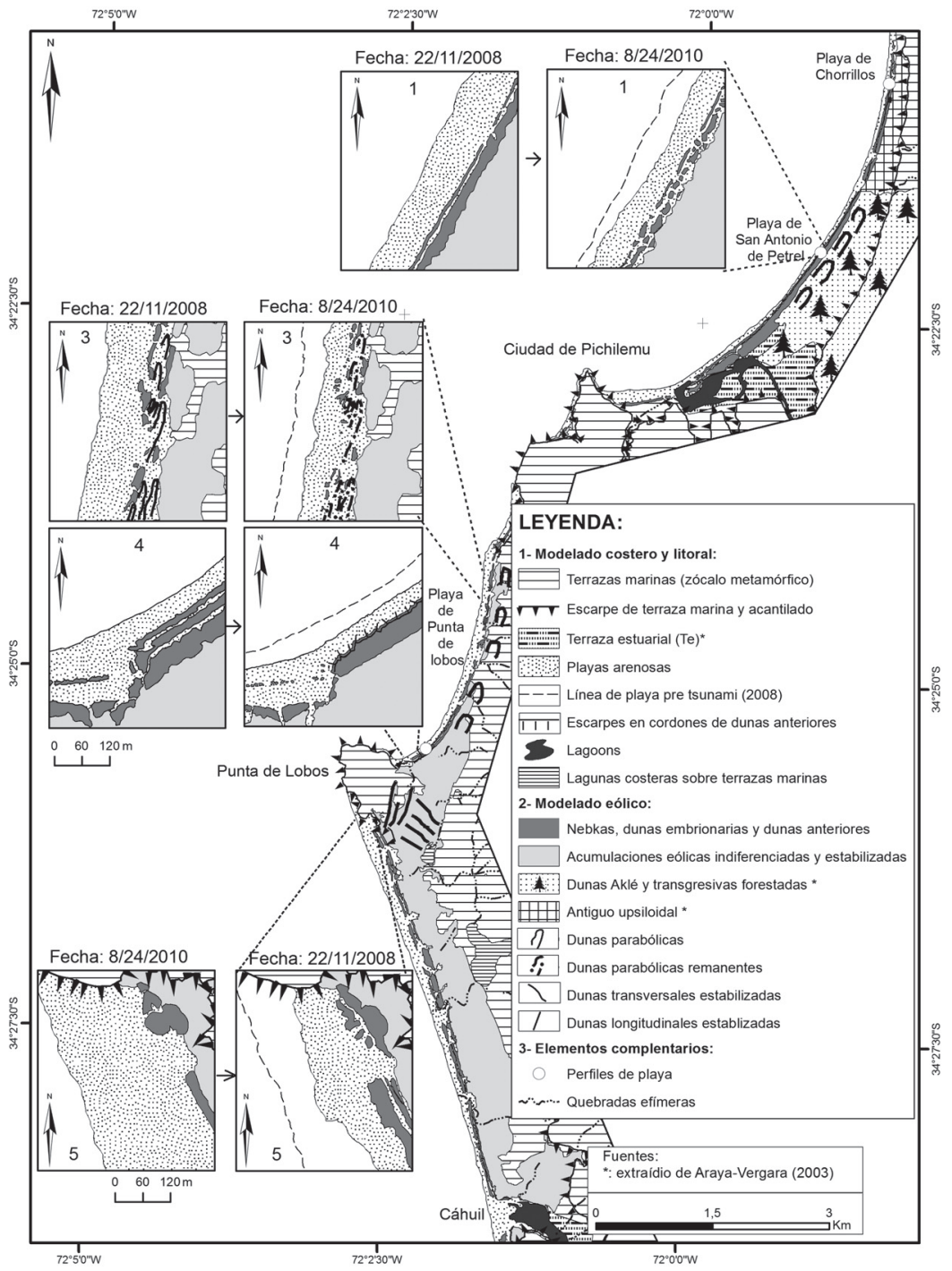

Fuente: Elaboración propia. 
dinales y transversales respectivamente, pero estabilizados por la vegetación. Estos sistemas no fueron alterados por el tsunami.

Otro efecto del tsunami fue identificado en los perfiles de playas y las dunas anteriores asociadas, tal como se graficó en la Figura $N^{\circ}$ 2. Debido a la existencia de perfiles de playa del año 2004, se pudo comparar con los realizados en 2012 (Figura $N^{\circ} 3$ ). De ello se establece que no hay evidencias de reconstrucción hasta el año 2012, solo en el sector de San Antonio, que corresponde a la sección media de la ensenada de Pichilemu. No obstante ello, solo permanecen como remanentes, los cordones de dunas anteriores de posición más interna (a sotavento de los primeros), principalmente en la zona distal de la bahía de Pichilemu (Chorrillos). De acuerdo a las observaciones del año 2004, había cordones de dunas con abundante cobertura de Carpobrotrus aequilaterus, que es una planta suculenta y rastrera que solo se desarrolla en áreas fuera del ataque del oleaje. La ausencia de esta planta posterior al tsunami fue la evidencia de la destrucción de los cordones de dunas. A su vez, la ausencia de dunas embrionarias de manera generalizada hasta febero de 2014, permitía también asumir la falta de alimentación general en la playa impidiendo su recontrucción.

Figura $\mathrm{N}^{\circ} 3$

Perfiles de playa de Chorrillos y San Antonio, sector distal y medio respectivamente de la bahía de Pichilemu, y Punta de Lobos (zona proximal). Los perfiles muestran diferencias notorias asociadas a la condición de ataque del oleaje, como también el efecto de la erosión postsunami. Se debe apreciar el retroceso significativo de la playa

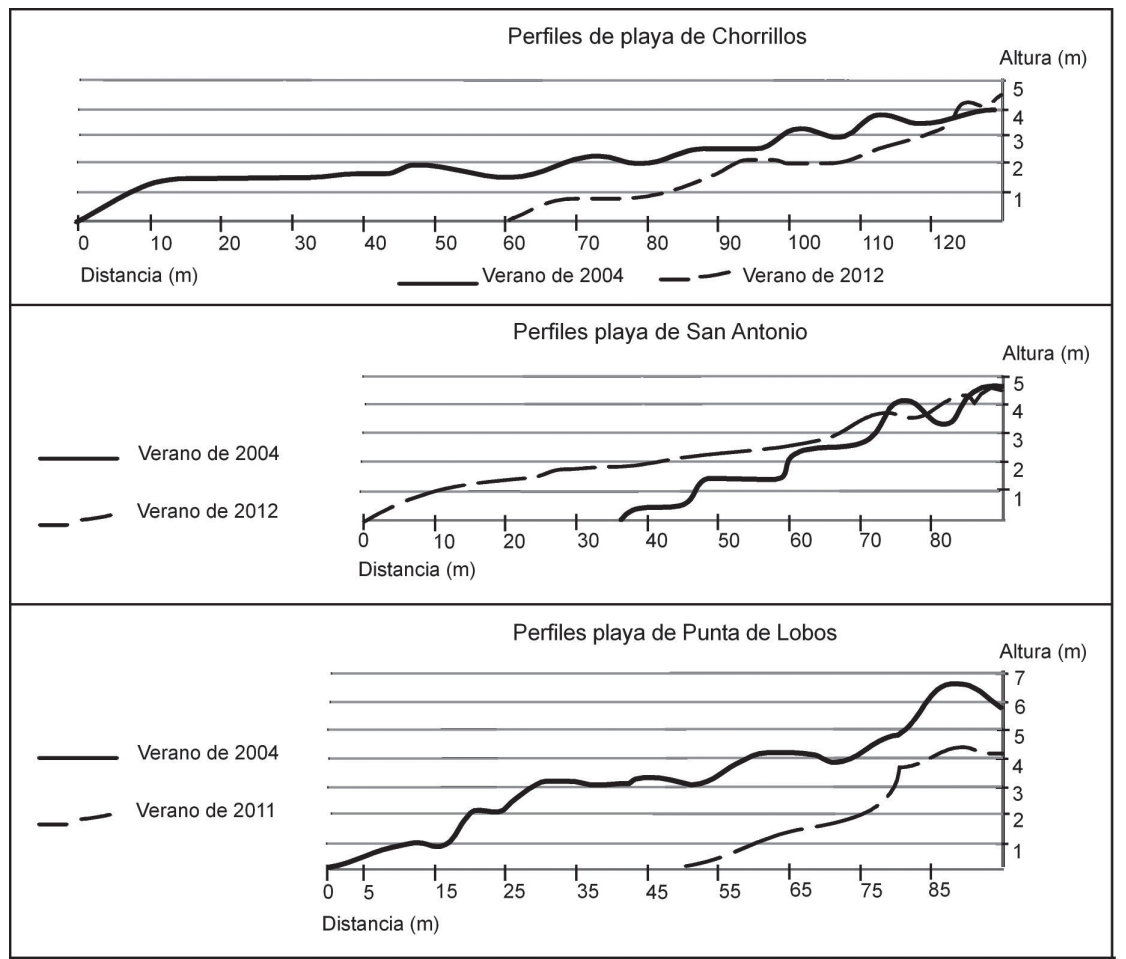

Fuente: Elaboración propia.

Al analizar los perfiles de playa del sector de la bahía de Pichilemu (2004 y 2012) se aprecia el predominio de perfiles multise- cuenciales, es decir de varias secuencias de playas (de ante y postplaya), que representan una condición de acresión sedimentaria. Sin 
embargo, en el caso de acresión relativa (Figura $N^{\circ} 3$, de San Antonio) esta podría estar asociado a un suministro de arenas suficiente como para lograr ensanchar la playa arenosa, pero no el necesario para generar dunas embrionarias; esta situación es aplicable a la parte distal, media y proximal de la ensenada de Pichilemu.

No obstante lo anterior, al año 2014, se puede observar un nuevo patrón en las playas. La Figura $N^{\circ} 4$ expresa los cambios posteriores al tsunami, en donde destaca el desmantelamiento de los cordones de dunas anteriores, sobre todo aquellos de la zona norte, localizados junto a la desembocadura del estero. Estos cordones fueron erosionados y su forma fue enmascarada existiendo hasta la actualidad un gran cordón, sin dunas embrionarias. Sin embargo, al analizar el ancho de la playa actual, se apercia que este ha aumentado, lo que indica una condición actual de acreción sedimentaria. Se debe destacar que la imagen de referencia del año 2014 es del mes de julio, es decir, durante el periodo en que existe más tendencia a la ocurrencia de playas con perfil de invierno, vale decir, de tipo erosional.

Figura $\mathrm{N}^{\circ} 4$

Cambios en la playa arenosa de Pichilemu, zona proximal. Líneas continuas indican línea de playa pretsunami (2008). Líneas segmentadas señalan localización de cordones de dunas anteriores.

Sector dos en figura uno
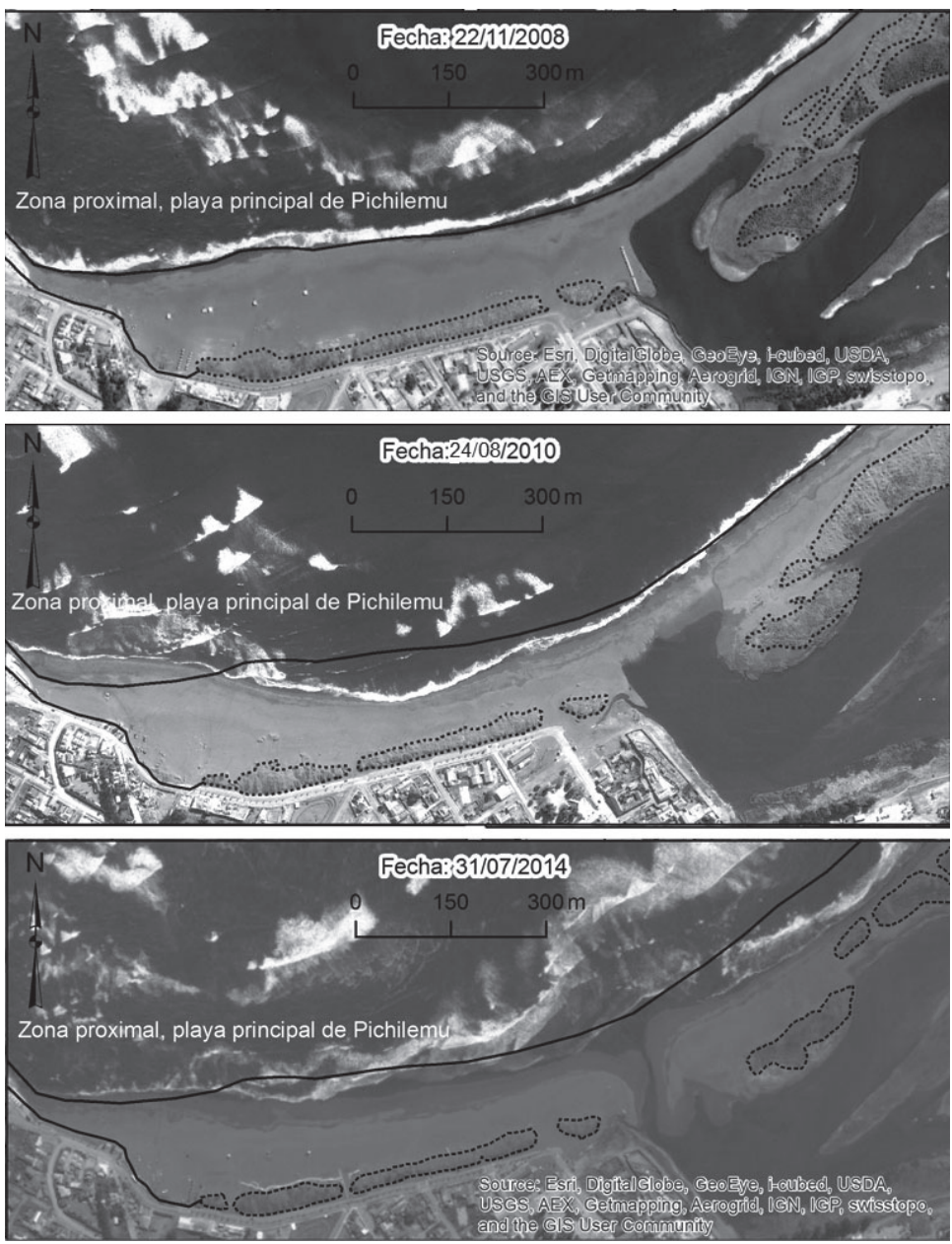

Fuente: Elaboración propia. 
En Punta de Lobos, los perfiles de playa realizados en la zona proximal y media de la ensenada durante el año 2004, presentaban una predominancia a la multisecuencialidad (Figura $\mathrm{N}^{\circ} 3$ ); al comparar estos perfiles con los de las condiciones de la playa postsunami, estos tienden a ser bisecuenciales y monosecuenciales, es decir que no solo son más estrechos, sino que están poco desarrollados (Figura $N^{\circ}$ 5). Cabe señalar que hasta mayo del 2013 los perfiles mantenían tal condición y que en enero de 2014 no se observaban dunas embrionarias. En la ensenada de Punta de Lobos, las dunas asociadas a la familia de las dunas anteriores (según el continuum dunar), presentaban una importante cobertura vegetal de Ammophila arenaria (observado en 2004). El impacto del tsunami en este sector de la bahía fue muy intenso, toda vez que el retroceso de la playa fue del orden de los 100 m y que este se ha mantenido por la acción del oleaje que ataca prácticamente en todo estado de marea el microacantilado en duna, (Figura $\mathrm{N}^{\circ} 5$ ), que continúa retrocediendo en la actualidad a través de procesos gravitacionales.

Figura $\mathrm{N}^{\circ} 5$

Playa Punta de Lobos, microacantilado en duna. La urbanización existente en el lugar tenía un cerco de deslinde a unos 100 m de la línea de máxima resaca, que ya no existe (marzo de 2012). Sector cuatro en figura uno. Fuente: colección personal de los autores

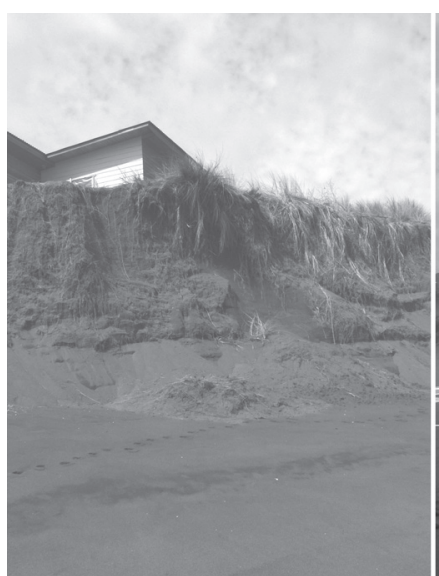

Fuente: Elaboración propia.

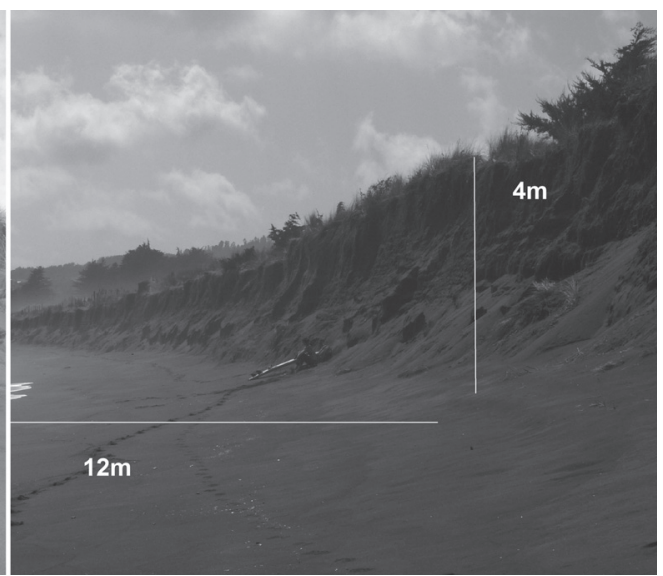

Figura $\mathrm{N}^{\circ} 6$

Cambios en la playa arenosa de Punta de Lobos, zona proximal. Líneas continuas indican línea de playa pretsunami (2008). Líneas segmentadas señalan localización de cordones de dunas anteriores. Sector cuatro en figura uno
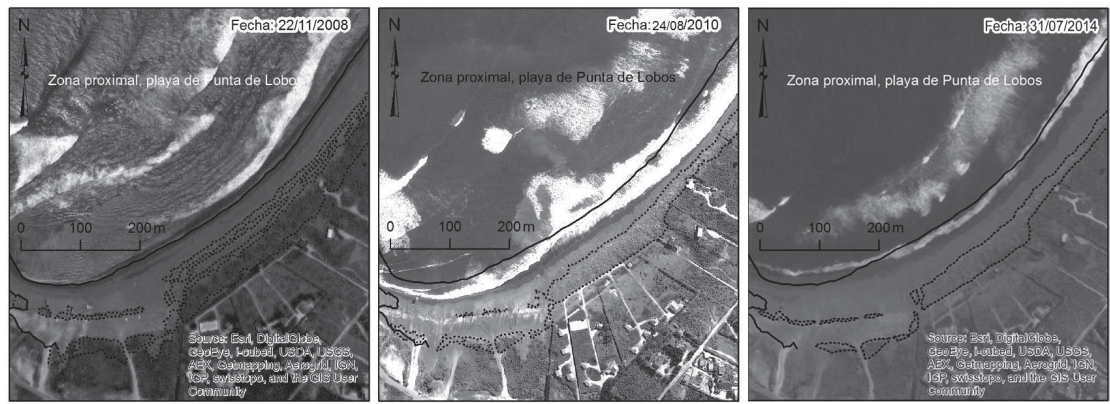

Fuente: Elaboración propia. 
El retroceso de la línea litoral en esta parte de la bahía se observa en las Figuras $\mathrm{N}^{\circ}$ 2 y $N^{\circ} 6$. En la sección proxinal de la bahía de Punta de Lobos, al que se asocia un prominente headland de rocas esquistosas, es donde se aprecia la mayor ausencia de abastecimiento hasta el año 2012-2013. Además, como documentado por Soto (2005) en este sector existían dunas anteriores que si bien sufrían el ataque del oleaje de invierno, eran posteriormente reconstruidas. No obstante, el crecimiento de la playa en el último año, este no ha sido lo suficientemente abudante como para generar dunas embrionarias. La condición de perfiles de playa erosionales se mantiene hasta la fecha.
En la zona media de la ensenada de Punta de Lobos, en enero de 2012 se había observado, aunque en un estado muy incipiente, la generación de microdunas (nebkas) asociadas a los obstáculos dejados por los restos de raíces de Ammophila arenaria que fueron arrancadas por el tsunami, desde los cordones de dunas existentes previamente; estas dunas embrionarias estarían conformando actualmente un incipiente cordón de dunas anteriores (Figura $N^{\circ} 7$ ). La relación evolutiva de estas dunas embrionarias está asociada a las familia de las dunas anteriores, en un sector que antes del tsunami poseía varios cordones de dunas anteriores, con dunas embrionarias y dunas parabólicas, todas formas indicativas

Figura $\mathrm{N}^{\circ} 7$

Formación de duna embrionarias a partir de raíces de Amophila arenaria remanente de cordones de dunas anteriores y parabólicas. Sector medio ensenada Punta de Lobos. La flecha indica una micro duna (enero de 2012)

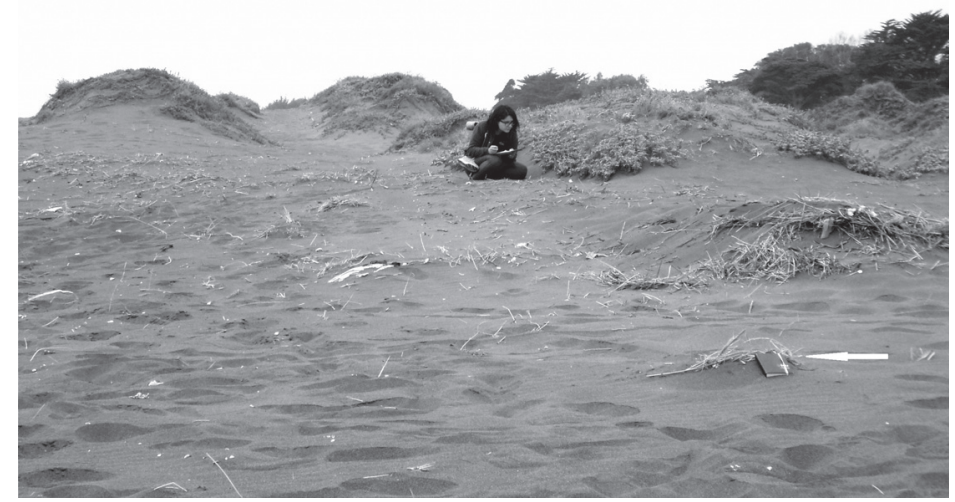

Fuente: Archivo personal de los autores.

del abastecimiento de arena. El perfil de la playa era de tipo mulsisecuencias acrecional.

El litoral de Cáhuil presenta una forma rectilínea, de orientación oblicua, perpendicular al oleaje indidente, y no está controlada por la presencia de un headland. Los cambios observados corresponden al retroceso de la playa arenosa y de los primeros cordones de dunas anteriores, donde además existe un pequeño campo de dunas en estado avanzado de estabilización. En este sector la pérdida de playa había generado hasta el periodo 20122013, una condición sostenida de retroceso ya que el oleaje atacaba directamente los cordones litorales remanentes, generando la erosión de estos y el ingreso de olas de tormenta y bravezas que alcanzaban hasta las dunas, contribuyendo a su erosión y potencial reactivación, a través de los corredores de deflación e incipientes blowout asociados.

El retroceso de la playa arenosa también era observable en la zona del lagoon y estuario de Cáhuil. Esta condición es especialmente importante ya que la barrera de Cáhuil se mantenía cerrada prácticamente durante todo el año, salvo en eventos excepcional- 
mente lluviosos. Esta condición se observa en la figura 8, a través del ancho de la barrera. Durante los años sin crecidas fluviales la barrera se abría artificialmente para favorecer la construcción de salinas que se desarrollan como actividad económica al interior del estuario. Desde el teremoto y tsunami la barrera ha mantenido su inlet en permanente fun- cionamiento. Tanto es así que en la figura ya citada es posible observar la acción del oleaje y las mareas en la parte interna del inlet, generando formas de washover fan. Si bien, en el último periodo se ha observado un considerable crecimiento del ancho de la playa en Cáhuil, aún no se logra alcanzar el límite anterior al terremoto y tsunami (Figura $N^{\circ} 8$ ).

Figura $\mathrm{N}^{\circ} 8$

Cambios en la playa arenosa de Cáhuil y desembocadura del estero Nilahue. Líneas continuas indican la posición de la línea de playa pre tsunami (2008). Líneas segmentadas señalan la localización de cordones de dunas anteriores. Sector seis en figura uno
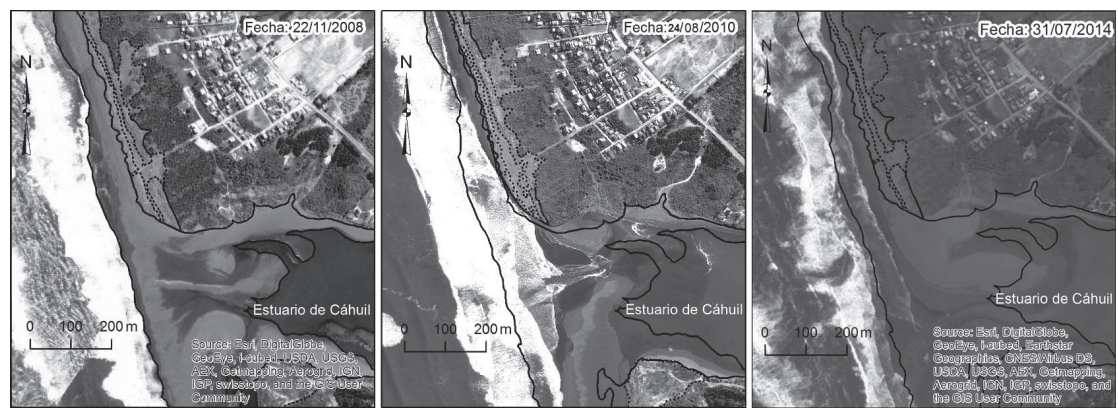

Fuente: Elaboración propia.

Si bien las figuras anteriores expresan graficamente, a través de las imágenes extraídas de Google Earth, el retroceso y posterior acreción de las playas arenosas, en la Figura $N^{\circ} 9$ se expresan tales pérdidas en términos porcentuales. En todo el litoral analizado hubo pérdida de la línea de costa que se mantuvo como una condición constante hasta el año 2014, cuando se pudo medir una condición de acreción, que aún no recupera la condición morfosedimentaria existente antes de terremoto/tsunami.

Para poder visualizar más ampliamente los cambios de la línea de costa, se realizaron mediciones para los años 2004 y 2007. Como resultado se obtuvo la condición dinámica de las playas, la que es diferencial en función de la posición en el sistema de bahías estructurales. El patrón espacial observado no es concordante con la dinámica de ensenadas que se observaba antes del 27 $F$, ya que los mayores espersores de dunas y cordones de dunas anteriores y ancho de perfiles de playa que existían en la zona dis- tal del sistema, en el sector de Chorrillos, se registra el menor incremento entre $2010 \mathrm{y}$ 2014, mientras que la zona proximal, Punta de Lobos y Cáhuil, son actualmente las áreas con mayor aumento del ancho de playa.

En Punta de Lobos, la estimación de pérdida de playa alcanzó el $42 \%$, valor muy expresivo de los fenómenos que ocurren en esa sección de la bahía, donde en el último año se ha registrado solo el incremento del ancho de la playa, alcanzando una amplitud cercana a la anterior, pero sin formación de dunas.

El litoral de Cáhuil corresponde a la sección de la bahía donde se han podido constatar los mayores impactos en cuanto a retroceso de la línea de costa, equivalente a un $51 \%$ (Figura $N^{\circ} 2, N^{\circ} 8$ y $N^{\circ} 9$ ), pero también el mayor crecimiento porcentual de la misma, aunque muy diferente al nivel anterior al evento. Esta parte del litoral presenta una forma rectilínea, de orientacion oblicua, perpendicular al oleaje incidente, y no está controlada por la presencia de un headland, 


\section{Figura $\mathrm{N}^{\circ} 9$}

Superficies de las playas en el sector comprendido entre Cáhuil y Pichilemu, correspondientes a los años 2004-2014, y variación porcentual entre los años 2008-2010 y 2010-2014

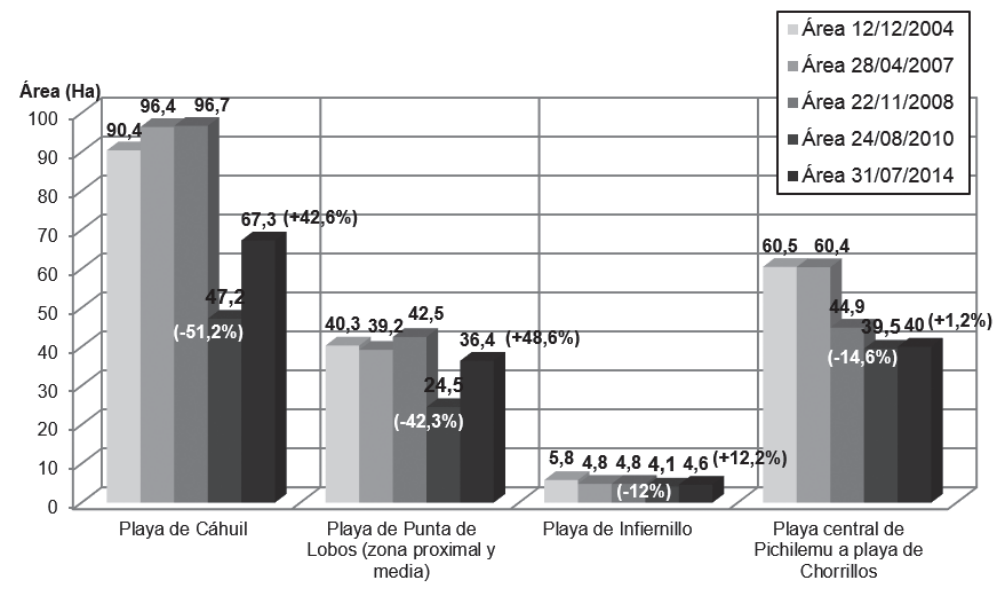

Fuente: Elaboración propia.

pero es el área del exhutorio de la cuenca aportante de sedimentos al litoral.

En la zona distal, en Chorrillos, Ilama la atención el bajo incremento del ancho de la playa, el que puede ser explicado por su posición distal en el sistema.

\section{Dinámica litoral}

Si bien la zona de rompiente es altamente variable, existen ciertos patrones que están asociados a la posición en la ensenada, a la estacionalidad y a las condiciones climáticoatmosféricas del área de estudio. En ese marco se analizan las observaciones puntuales de diferentes fechas, desde 1962 a julio de 2014.

El análisis de la dinámica de la zona de rompiente muestra un predominio de playas de carácter intermedio, tanto en la ensenada de Pichilemu como en Punta de Lobos (Figura $\left.N^{\circ} 10\right)$. En Pichilemu, a pesar de la variabilidad encontrada se aprecia un predominio de rompientes intermedias de tipo barra y surco longitudinal (Bar Lonfshore Trougth, LBT), tanto en la zona media como distal, habiendo condiciones más reflectivas solo en la parte proximal. Lo observado en marzo de 2011 y enero de 2012, indica un marcado predominio de condiciones de "playa disipativa" que debieran obedecer a situaciones estacionales.
La última observación de 2014, en imagen de invierno, entrega un patrón propio de ensenadas estructurales: playa reflectiva, intermedia y disipativa en la zona proximal, media y distal respectivamente.

En Punta Lobos, el efecto del prominente headland influye en la marcada refracción de los trenes de olas en la parte proximal de la ensenada, condicionando no obstante una playa intermedia, donde las formas de barra y surco longitudinal (Bar Lonfshore Trougth, LBT) y playa y barra rítmica (Beach Bar Rhytmic, RBB) son predominantes. Las condiciones observadas en marzo de 2011, son de una playa disipativa en la parte proximal (de alta energía, con olas de más de $3 \mathrm{~m}$ ), y de barra y surco longitudinal (Bar Lonfshore Trougth, LBT) en la parte media, situaciones que se mantienen en enero de 2013. En 2014, se mantiene este patrón. El litoral de Cáhuil, por su parte, presenta playas del tipo intermedias y disipativas.

Si se analizan las bahías como un sistema, en la localización de los tipos de playas existen patrones generales, tales como que la zona de Cáhuil por su posición proximal, recibe los aportes sedimentarios del estero Nilahue y es área de transferencia hacia el norte, asimismo, la zona de Punta Lobos, también es un área de transferencia debido al 
Figura $\mathrm{N}^{\circ} 10$

Playas dominadas por olas; Pichilemu, Punta de Lobos y Cáhuil. Actualizado de Soto (2005)

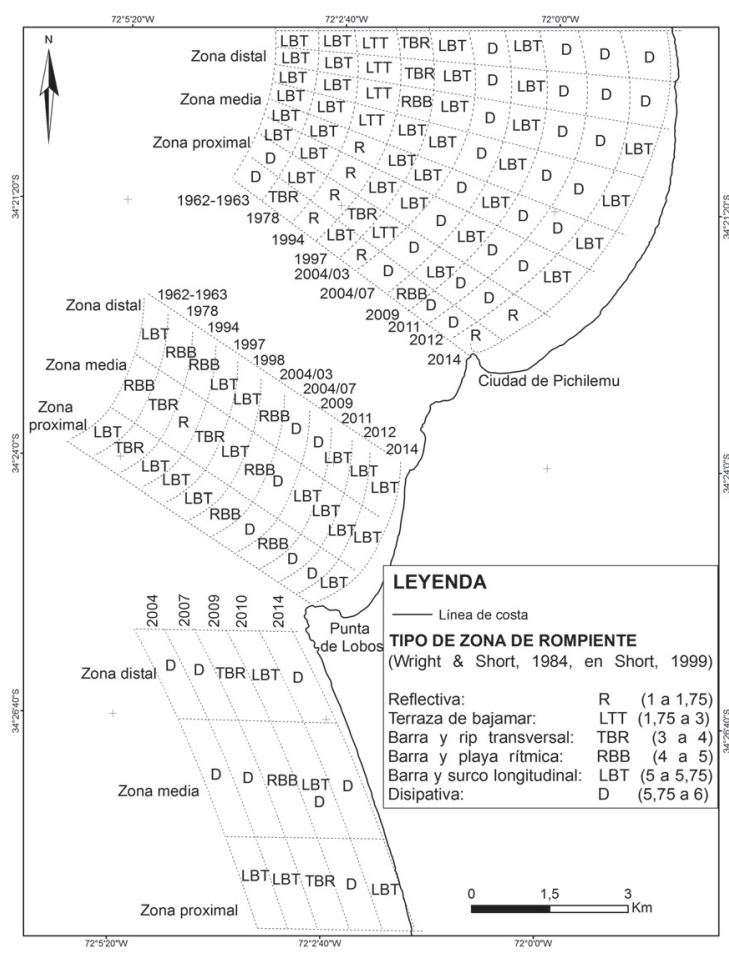

Fuente: Elaboración propia.

efecto en la refracción del oleaje que ejerce el headland, mientras la bahía de Pichilemu es la ensenada receptora, pero de manera relativa, ya que hacia la parte distal, fuera del área de estudio, existe un litoral rectilíneo que contribuye a la transferencia de masa deriva abajo.

Por otro lado, este proceso de transferencia sedimentaria, que permite visualizar los patrones ya señalados, no permite identificar el impacto asociado a la subsidencia medida para el sector, pues la escala de análisis empleada, espacial y sobre todo temporal de observación, no lo permite.

\section{Tendencias de cambio}

A partir de un escenario litoral controlado por la estructura (Araya-Vergara, 1983, 1985, 2003), en que la transferencia de masa está a su vez influida por las salientes rocosas y la forma del litoral, correspondiendo a un sistema de ensenadas estructurales (Soto, 2005), se analizan las condiciones geodinámicas asociadas a los impactos cosísmicos del terremoto de 2010, es decir, el tsunami y la subsidencia costera local en $50 \mathrm{~cm}$, documentados por Farías et al. (2010, 2011), Quezada et al. (2010, 2012) y Vargas et al. (2011).

Observaciones realizadas en terreno desde al año 2011-2013, evidencian que las playas arenosas sufrieron una fuerte erosión y retroceso, y que no hubo un inmediato proceso de alimentación y acreción de estas, salvo lo analizado en imágenes Google Earth de julio de 2014. Si bien se ha observado acreción de las playas, la presencia de escarpes en las dunas y la ausencia de dunas embrionarias demuestran que la erosión de playas es aún relevante y que el abastecimiento de arenas aún no alcanza las condicciones de antes de la subsidencia costera y la erosión por el tsusami. Dicha condición de erosión fue 
observada por Ibarra (2013) en las playas y dunas anteriores de Cáhuil. A este respecto es importantes señalar que el proceso de formación de dunas anteriores y sobre todo de microdunas, tenía una recurrencia estacional. Soto (2005) registró fotográficamente la destrucción de invierno y la construcción de verano de microdunas.

Los procesos de evolución morfológica postsunami fueron también analizados por Villagrán et al. (2013) en la flecha litoral del sistema estuarial del río Mataquito $\left(34^{\circ} 52^{\prime} \mathrm{S}\right.$, $\left.72^{\circ} 09^{\prime} \mathrm{W}\right)$ y en las playas de Duao $\left(34^{\circ} 53^{\prime} \mathrm{S}\right.$, $\left.72^{\circ} 10^{\prime} \mathrm{W}\right)$, asociadas también a la subsidencia cosísmica del mismo terremoto y tsunami. Los autores señalan que si bien la flecha litoral fue fuertemente erosionada, fue rápidamente reconstruida producto de un favorable abastecimiento sedimentario, probablemente asociado al río Maule y al campo de dunas de Putú-La Trinchera, sumado a descargas sedimentarias del río Mataquito. Esta evolución positiva no ha sido observada en el área de Pichilemu, a pesar que podrían ser comparables ya que ambos sectores están muy cercanos y presentan una morfología de ensenadas; las diferencias pueden se entendidas por subsidencia cosísmica, menos aporte sedimentario, solo atribuido al estero Nilahue y el ángulo de incidencia del oleaje en el sistema de ensenadas.

Komar (2010) señala que la erosión sostenida es el proceso característico de costas afectadas por subsidencia, tal como analizado en el litoral de Nueva Zelanda afectado por subducción cosísmica de hasta $2 \mathrm{~m}$ en 1931, en donde el retroceso de la playa fue el rasgo evolutivo distintivo en estas ensenadas.

También es imporante relacionar los resultados anteriormente expuestos con aquellos obtenidos por Tanaka et al. (2012) en el sistema litoral de Miyagui, pues también se observaron fuertes cambios morfológicos producto de la susbidencia cosísmica y tsunami por el terremoto del 11 de marzo del 2011 del este de Japón. Los autores destacan el fuerte retroceso y segmentación de las playas, de los sistemas dunares, de las barreras, flechas e inlets, como también los cambios en los perfiles hidráulicos de los lagoons analizados. Ibarra (2013) evidenció cambios similares en la desembocadura y lagoon del estero Nilahue, en Cáhuil.
La remoción de las arenas litorales por un tsunami y su posterior retrabajo, generan cambios muy dinámicos en la morfología litoral (Quezada et al., 2012). En tal sentido, la teoría acumulada indica que las olas de tsunami producen respuestas erosivas y depositacionales diferenciales en los sistemas litorales y que los procesos de subsidencia tectónica tienden a la erosión de las costas (Bird, 2008; Davidson-Arnott, 2009) lo cual es análogo a los resultados obtenidos por los autores anteriormente citados y a los de la presente investigación.

Sin embargo, de acuerdo a Tanaka et al. (2012), para el caso de los tsunamis, los procesos de restauración en los sistemas estuariales y costeros dependen mucho del régimen hidrodinámico y de la disponibilidad de abastecimiento sedimentario, siendo dos factores que regulan la posibilidad que las playas recuperen su forma original, a los cuales puede agregarse el efecto que tienen las salientes rocosas y la forma del litoral en la transferencia de sedimentos (Soto, 2005). El hecho que las arenas removidas por un tsunami quedan sueltas con poca compactación, de manera que el oleaje sucesivo, especialmente luego de marejadas, puede volver a depositarlas, contribuye a la acreción litoral, tal como fue observado por Quezada et al. (2012) en la zona litoral del Mataquito y Bucalemu (Chile central). No obstante, esta dinámica no ha sido identificada en Pichilemu.

En el tramo litoral Cáhuil-Pichilemu existen algunas evidencias geomorfológicas muy locales pero significativas que podrían estar evidenciando una condición de transferencia de masa en las parte medias y distales de las ensenadas de Pichilemu y Punta de Lobos (Figura $N^{\circ}$ 9). En el primer caso se trata de perfiles de playa multisecuenciales y en el segundo, de microdunas, en los sectores en que se identificó acresión de playas en la imagen del año 2014.

La dinámica de la zona de rompiente da cuenta de escasos cambios en el tipo de playa, ya que se mantienen los tipos intermedios como formas predominantes, sin que se hayan observado cambios en la zona de surf debidos a la condición de subsidencia del litoral, pero ello puede deberse a que las observaciones realizadas no tuvieron una 
recurrencia temporal y por lo tanto esta información no puede ser considerada como concluyente.

Otro antecedente considerado es la presencia de una terraza estuarial, Te y la incisión del lecho meandrante del estero San Antonio de Petrel, que Araya-Vergara (2003) estableció como una evidencia genética de un solevantamiento más acelerado que el ascenso del nivel del mar. No obstante su posición, estas terrazas en el sector de Cáhuil y en el estero Nilahue, evidencian la subsidencia ya que fueron inundadas, situación que se mantiene hasta la fecha.

En este contexto, se plantea que se ha producido un significativo impacto asociado a la subducción de la línea de costa en la dinámica costera en estas bahías controladas por la estructura, con una marcada influencia del headland de Punta de Lobos y de Pichilemu en la acción del oleaje. Basados en la teoría de las playas de medio corazón de Silvester (1960, en Araya-Vergara, 1983), o headland bay beachs, se podría señalar que existiría una tendencia a acentuar la función del headlands rocoso, aumentando la condición de refracción del oleaje, lo cual Ilevaría a generar una condición de ataque del oleaje mayor y por lo tanto, acentuando la curvatura de la bahía. Esto es consecuente con la situación observada hasta el año 2013, pero a partir del año 2014, se registra una condición de acreción de las playas que permite plantear una respuesta positiva y adaptación sedimentaria similar al estado anterior a 2010. Esta nueva condición debe ser observada en los siguientes años para poder establecer si es la nueva tendencia evolutiva, o bien obedece a una variación estacional.

\section{Conclusiones}

En relación a las bahías estructurales o headland bay beach, estas deben ser consideradas como sistemas costeros complejos de morfogénesis estructural y con una condición geodinámica actual, relacionada a la acción diferencial del oleaje y en consecuencia, altamente sensibles a los cambios que ocurren en el sistema.

A partir de los antecedentes previos disponibles, que comprenden el período 2004-
2008 y 2010-2014, se puede concluir que el terremoto y el tsunami ejercieron un gran impacto en el litoral generando la condición de erosión extrema en las playas arenosas, lo que produjo una nueva condición basal, que se tradujo en la falta de aporte sedimentario a las playas. El tsunami desmanteló, destruyó y erosionó las playas y dunas, estas no se han reconstruido en casi cuatro años. Durante el último año sin embargo, se aprecia un cambio reflejado en la acreción de las playas arenosas.

El reconocimiento de perfiles multisecuenciales y microdunas, muy localizadas, constituyen las evidencias concretas de un nuevo estado de equilibrio asociado a la alimentación incipiente y diferencial de arena a la playa. Esta situación requiere de observaciones sistemáticas futuras que permitan discriminar situaciones estacionales de tendencias de cambio efectivas.

El efecto cosísmico del terremoto y tsunami de 2010 generó nuevas condiciones geodinámicas en las playas arenosas en el sistema de ensenadas estructurales de Pichilemu, asociadas a la erosión sostenida hasta el año 2013 debido a la falta de aporte sedimentario, propio de los ambientes de subsidencia; a partir del año 2014, un nuevo estado geodinámico asociado a la llegada de sedimentos a las playas registrado gracias a la acreción generalizada de la línea de costa. El abastecimiento de arenas no ha sido tan abundante como para desarrollar cordones de dunas anteriores o microdunas, existentes antes del terremoto y tsunami.

\section{Referencias bibliográficas}

AAGAARD, T. \& MASSELINK, G. The Surf Zone. In: SHORT, A.D. Handbook of Beach and Shoreface Morphodynamics. Chichester: John Wiley \& Sons, Ltd, 1999, p. 72-118.

AAGARD, T.; DAVIDSON-ARNOTT, R.; GREENDWOOD, B. \& NIELSEN, J. Sediment supply from shoreface to dunes: Linking sediment transport measurements and long-term morphological evolution. Geomorphology, 2004, No 60, p. 205-244.

AAGARD, T. \& HUGHES, M. Sediment suspension and turbulence in the swash 
zone of dissipative beaches, Marine Geology, 2006, Vol. 228, N 1-4, p. 117-135.

ARAYA-VERGARA, J.F. Influencias morfogéneticas de los desalineamientos y líneas de costa contrapuestas en el litoral de Chile Central. Informaciones Geográficas, 1983, Nº 30, p. 3-23.

ARAYA-VERGARA, J.F. Trend analysis of shoreline changes and coastal management in Central Chile (33-34.5으. In: Actes EscursionSymposium No 9: La Cöte Atlantique. Paris: Union Géographique Internationale, Commission sur I'Environnement Cötier, 1985, p. 99-110.

ARAYA-VERGARA, J.F. Cambios actuales de la línea litoral en Chile central sur. Revista Geográfica de Chile Terra Australis, 1986, № 29, p. 23-28.

ARAYA-VERGARA, J.F. Sistema de interacción oleaje-playa frente a los ergs de Chanco y Arauco, Chile. Cayana Oceanol, 1996, Vol. 4, No2, p. 159-167.

ARAYA-VERGARA, J.F. Génesis de la ensenada en zeta de Pichilemu, Chile Central. Investigaciones Geográficas, 2003, N³7, p. 41-65.

ARRIAGADA, J. Geomorfología comparada en la zona semiárida de Chile: casos Copiapó y Choapa. Santiago de Chile: Tesis para optar al grado de Magíster en Geografía, Universidad de Chile, 2009

ARRIAGADA, J.; SOTO, M-V. \& SARRICOLEA, P. Morphodynamic environment in a semiarid mouth river complex. Choapa River, Chile. In: MARGHANY, M. (editor). Advanced Geociences Remote Sensing. Croatia, 2014, p. 253-271.

BENEDET, L.; KLEIN, A. \& HSU, J.R.C. Practical insights and applicability of empirical bay shape equations. Coastal Engineering, 2004, p. 2181-2193.

BIRD, E. Coastal Morphology, An Introduction. Chichester: Wiley \& Sons, 2000.

BIRD, E. Coastal Morphology, An Introduction. Chichester: Wiley \& Sons, 2008.
BRANDER, R.W. \& SHORT, A.D. Morphodynamics of a large-scale rip current system at Muriwai Beach, New Zealand. Revista Marine Geology, 2000, № 165, p. 27-39.

BRÜGGEN, J. Fundamentos de la Geología de Chile. Santiago de Chile: Editorial Instituto Geográfico Militar (IGM), 1950.

CASTILLA, J.C. Earthquakes caused coast uplift and its efects on rockies intertidal help comiunites. Science, 1988, № 242, p. 440-442.

CASTILLA, J.C.; MANRÍQUEZ, P.H. \& CAMAÑO, A. Rocky shore coseismic ulplift and the 2010 mega earthquake in Chile. Marine Ecology Progress Series, 2010, № 418, p. 17-23.

COMTE, D.; EISENBERG, A.; LORCA, E.; PARDO, M.; PONCE, L.; SARAGONI, R.; SINHG, S.K. \& SUÁREZ, G. The 1985 central Chile earthquake: A repeat of previous great earthquakes in the region? Science, 1986, $\mathrm{N}^{\circ}$ 233, p. 393-500.

CECIONI, C. Esquema de Paleogeografía Chilena. Santiago de Chile: Editorial Universitaria, 1970.

ELGAR, S.; RAUBENHEIMER, B. \& GUZA, R.T. Current meter performance in a surf zone. Journal of Atmospheric and Aceanic Technology, 2001, № 18, p. 1735-1746.

EMERY, K.O. A simple method of measuring beach profiles. Limnology and Oceanography, 1961, №6, p. 90-93.

DAVIDSON-ARNOTT, R. Introduction to coastal process and Geomorphology. New York: Cambridge University Press, 2009.

DAVIS, R.A. Beach profiles. In: Encyclopedia of Beaches and Coastal Environments. Pennsylvania: Hutschinson Ross Publications, 1982.

FARÍAS, M.; VARGAS, G.; TASSARA, A.; CARRETIER, S.; BAIZE, S.; MELNICK, D. \& BATAILLE, K. Land-Level Changes Produced by the Mw 8.82010 Chilean Earthquake. Science, Vol. 329, №5994, p. 916-916.

FARÍAS, M.; COMTE, D.; ROECKER, S.; CARRIZO, D. \& PARDO, M. Crustal exten- 
sional faulting triggered by the 2010 Chilean earthquake: The Pichilemu Seismic Sequence. Tectonics, 2011, № 30, p. 1-11.

GONZÁLEZ, N. Características sismotectónicas de la brecha sísmica de PichilemuConstitución: Segmento sur no activado de la zona de ruptura del gran terremoto de 1906. Santiago de Chile: Memoria de título, Departamento de Ingeniería Civil, Universidad de Chile, 1997.

HSU, J.R.C \& EVANS, C. Parabolic Bay Shapes and Aplications. Institution of Civil Engineers, 1989, Vol. 87, № 2, p. 556-570.

IBARRA, C. Geomorfología aplicada en la evaluación y análisis de amenazas naturales en la zona sur de la comuna de Pichilemu: sectores de Punta de Lobos-Cáhuil-Estero Nilahue. VI Región, Chile. Santiago de Chile: Memoria para optar al título profesional de Geógrafo, Universidad de Chile, 2013.

LAGOS, M.; ARCAS, D.; RAMÍREZ, T.; SEVERINO, R. y GARCíA, C. Alturas de tsunami modeladas y observadas. Evento del 27 de febrero del 2010, Chile. Resultados preliminares. Santiago de Chile: Instituto de Geografía, Pontificia Universidad Católica de Chile, 2010.

KLEIN, A.H.F. \& DE MENEZES, J.T. Beach morphodinamics and profile sequence for a headland bay coast. Journal of Coastal Research, 2001, Vol. 17, № 4, p. 812-835.

KLEIN, A.H.F.; ANDRIANI, J.N. \& DE MENEZES, J.T. Shoreline Salients and Tombolos on the Santa Catarina coast (Brazil): description and analysis of the morphologycal relationships. Journal of Coastal Research, 2002, №36, p. 425-440.

KLEIN, A.H.F.; FILHO, L. \& SCHUMACHER, H. Short-term beach rotation processes in distinct headland bay beach systems. Journal of Coastal Reseach, 2002b, Vol. 18, № 3, p. 442-458.

KLEIN, A.H.K.; FERREIRA, O.; DIAS, J.M.A.; TESSLER, M.G.; SILVEIRA, L.F.; BENEDET, L.; DE MENEZES, J.T. \& ABREU, J.G.N. Morphodynamics of structurally controlled headland-bay beaches in southeastern Brazil:
A review. Coastal Engineering, 2010, № 57, p. $98-111$.

KOMAR, P.D. Shoreline Evolution and Management of Hawke's Bay, New Zealand: Tectonics, Coastal Processes, and Human Impacts. Journal of Coastal Research, 2010, № 26, p. 143-156.

KRUMBEIN, W.C. Shore processes and beach characteristics. Beach Erosion Board Tech., 1947, № 3, p. 1-38.

LAVALLE, P.D. \& LAKHAN, V.C. A spatialtemporal analysis of the development of a long-spiral shaped embayment. Earth Surface Processes and landforms, 1997, $\mathrm{N}^{\circ} 22$, p. 657-667.

LAGOS, M. Zonificación del riesgo de tsunami en el centro-sur de Chile. Revista de Geografía Norte Grande, 2012, № 53, p. 7-21.

LE BLOND, P. An explanation of the logarithmic spiral plan shape of headland-bay beaches. Journal of Sedimentary Petrology, 1979, Vol. 49, N 4, p. 1093-1100.

LE ROUX, J.P.; OLIVARES, M.D.; NIELSE, N.S.; SMITH, N.D.; MIDDLETOM, H.; FENNER, J. \& SSHMAN, S.E. Bay sedimentation as controlled bay regional crustal behaviour, local tectonics and eustatic sea-level changes: Coquimbo Formations (Miocene-Pliocene), Bay of Tongoy, central Chile. Sedimentary Geology, 2006, №184, p. 133-153.

MARTÍNEZ, C. El efecto de ensenada en los procesos litorales de las ensenadas de Valparaíso, Algarrobo y Cartagena, Chile Central. Santiago de Chile: Tesis para optar al grado de magíster en Geografía, Universidad de Chile, 2001.

MARTíNEZ, C.; QUEZADA, M. \& RUBIO, P. Historical changes in the shoreline and littoral processes on a headland bay beach in central Chile. Geomorphology, 2011, № 135 , p. 80-96.

MASSELINK, G. Alongshore variation in beach cups morphology in a coastal embayment. Earth Surface Processes and Landforms, 1999, No 24, p. 335-347. 
MASSELINK, G.; EVANS, T.; HUGHES, D. \& RUSSELL, P. Suspended sediment transport in the swash zone of a dissipative beach. Marine Geology, 2005, №216, p. 169-189.

NISHENKO, S.P. Circum-pacific seismic potential: 1989-1999. Pure and applied geophysics, 1991, No 135, p. 169-259.

QUEZADA, J.; JAQUE, E.; BELMONTE, A.; FERNÁNDEZ, F.; VÁSQUEZ, D. y MARTÍNEZ, C. Movimientos cosísmicos verticales y cambios geomorfológicos generados durante el terremoto de $\mathrm{Mw}=8,8$ del 27 de febrero del 2010 en el centro sur de Chile. Revista Geográfica del Sur, 2010, №2, p. 11-45.

QUEZADA, J.; JAQUE, E.; FERNÁNDEZ, A. y VÁSQUEZ, D. Cambios en el relieve generados como consecuencia del terremoto $M w=8,8$ del 27 de febrero del 2010 en el centro-sur de Chile. Revista de Geografía Norte Grande, 2012, №53, p. 35-55.

RUEGG, J.C.; RUDLOFF, A.; VIGNY, C.; MADARIAGA, R.; DE CHABALIERA, J.B.; CAMPOS, J.; KAUSEL, E.; BARRIENTOS, S. y DIMITROV, D. Interseismic strain accumulation measured by GPS in the seismic gap between Constitución and Concepción in Chile. Physics of the Earth and Planetary Interiors, 2009, No 175, p. 78-85.

SHORT, A.D. Waves-dominated beachs. In: SHORT, A.D. Handbook of Beach and Shoreface Morphodynamics. Chichester: John Wiley \& Sons, Ltd., 1999, p. 173-191.

SHORT, A.D. \& MASSELNIK, G. Embayed and structurally controlled beach. In: SHORT, A.D. Handbook of Beach and Shoreface Morphodynamics. Chichester: John Wiley \& Sons, Ltd., 1999, p. 204-229.

SHORT, A.D. Beaches of the Western Australian Coast: Eucla to Roebuck Bay. Sydney: Sydney University Press, 2005.

SIMONE, L.P. \& DE FALCO, G. Morphodynamics of a nontidal embayed beach: The case of Is Arutas (Western Mediterranean). Journal of Coastal Research, 2013, Vol. 29, Nº 6a, p. 63-71.
SOTO, M.V. Aspectos morfodinámicos de ensenadas desalineadas del litoral de Chile central: Pichilemu y caleta Los Piures. Revista de Geografía Norte Grande, 2005, № 33, p. 73-87.

SOTO, M.V. \& ARRIAGADA, J. Características dinámicas de ensenadas estructurales de Chile central. Maitencillo-Cachagua y Papudo, Región de Valparaíso. Revista de Geografía Norte Grande, 2007, №38, p. 99- 112.

SOTO, M.V.; ARRIAGADA, J.; CASTRO, C.P.; MÄRKER, M. y RODOLFI. G. Aspectos geodinámicos de un paleoestuario del desierto marginal de Chile: río Copiapó. Revista de Geografía Norte Grande, 2010, №46, p. 123-135.

SWEERS, K.B. Equilibrium Bays. A numerical study after the behaviour of equilibrium bays. Delft: Department Hydraulic Engineering, Faculty of Civil Engineering and Geosciences, Delft University of Technology, 1999.

TANAKA, H.; XUAN TINH, N.; UMEDA, M.; HIRAO, R.; PRADJOKO, E.; MANO, A. \& UDO, K. Coastal and estuarine morphology changes induced bye the 2011 great east Japan earthquake tsunami. Coastal Engineering Journal, 2012, Nº 54.

VARGAS, G.; FARÍAS, M.; CARRETIER, S.; TASSARA, A.; BAIZE, S. \& MELNICK, D. Coastal uplift and tsunami effects associated to the $2010 \mathrm{Mw}$ 8,8 Maule earthquake in Central Chile. Revista Andean Geology, 2011, No 38, p. 219-238.

VILLAGRÁN, C.P. Dinámica costera en el sistema de bahías comprendidas entre ensenada Los Choros y bahía Tongoy. Santiago de Chile: Memoria para optar al título de Geógrafa, Departamento de Geografía, Universidad de Chile, 2007.

VILLAGRÁN, M.; CIENFUEGOS, R.; CATALÁN, R. \& ALMAR, R. Morphological response of central Chile Sandy beaches to the 8.8 Mw 2010 earthquake and tsunami. In: 7th International Conference on Coastal Dynamics Arcachon, France, 2013.

YASSO, W. Plan geometry of headlandbay beaches. Journal of Geology, 1965, No 73, p. 702-714. 
